\title{
The Clinical Utility of the Diagnostic Criteria for Psychosomatic Research: A Review of Studies
}

\author{
Piero Porcelli ${ }^{a} \quad$ Jenny Guidi ${ }^{b}$ \\ ${ }^{a}$ Clinical Psychology Unit, IRCCS De Bellis Hospital, Castellana Grotte, and bepartment of Psychology, University of \\ Bologna, Bologna, Italy
}

\section{Key Words}

Clinical utility · Diagnostic Criteria for Psychosomatic Research · Psychosomatic medicine · Somatization · Illness behavior - Demoralization - Depression - Alexithymia . Irritable mood

\begin{abstract}
Background: The Diagnostic Criteria for Psychosomatic Research (DCPR) are a set of 12 psychosomatic syndromes and are provided with a reliable diagnostic structured interview. The DCPR have been proposed 20 years ago as an integrative assessment strategy that supplements the traditional psychiatric nosography for identifying patients within a given illness population whose psychosocial factors have clinical significance. This paper reviews their clinical utility, conceived as the degree and the amount of influence that the instrument has on multiple decisions and outcomes in clinical practice. Methods: Published reports which involved the use of the DCPR were identified by searching electronic databases. Studies which best displayed the clinical utility of the DCPR system were then selected and reviewed. Results: The DCPR system showed its clinical utility regarding the following clinical issues: (1) subtyping medical patients, (2) identifying subthreshold or undetected syndromes, (3) eval-
\end{abstract}

uating the burden of somatic syndromes, and (4) predicting treatment outcomes and identifying risk factors. Conclusions: The DCPR may help clinicians during the assessment process to recognize clinical conditions underlying symptom presentation, with important therapeutic and prognostic implications.

(c) 2015 S. Karger AG, Basel

\section{Introduction}

In any field of medicine and clinical psychology, including psychosomatic medicine, the effectiveness of the diagnostic process increases to the extent that it achieves three interrelated purposes, namely enhanced knowledge (providing clinicians with a meaningful framework that recognizes the underlying clinical condition beyond the presentation of symptoms, and identifying potential or unrecognized problems), enhanced communication (facilitation of communication among clinicians), and clinical utility (enhancement of decision making to improve the patient's health status) [1]. In particular, clinical utility refers to the degree and the amount of influence that the instrument has on multiple decisions and outcomes in clinical practice $[2,3]$. Concepts included in the con-

\section{KARGER 125}

(c) 2015 S. Karger AG, Base

0033-3190/15/0845-0265\$39.50/0

E-Mail karger@karger.com

www.karger.com/pps
Piero Porcelli

UO Psicologia Clinica

IRCCS Ospedale De Bellis, Via Turi 27

IT-70013 Castellana Grotte (Italy)

E-Mail piero.porcelli@irccsdebellis.it 
struct of clinical utility are therefore related not only to the classification, but also to the monitoring and predicting of symptom change and treatment outcome [4].

Although the new DSM-5 classification of somatic symptom and related disorders has introduced substantial modifications in diagnostic criteria, it does not seem to meet the basic requirements of clinical utility in the field of psychosomatic medicine and in the identification of the psychological factors influencing the course of medical disorders [5-8].

A change of strategy in the assessment of psychosocial factors associated with medical disorders would imply the need of accounting for two complementary aspects. At a clinical level, clinicians need to know more what kind of a person their patient is rather than what kind of disorder their patient has, given that a medical diagnosis and an appropriate medical follow-up is provided. At a research level, researchers need to work on classes of phenomena as homogeneously as possible to avoid mixing up apples with oranges and to add scientific knowledge to the role of psychosocial factors in the onset, course, prognosis, and treatment of medical illnesses.

The Diagnostic Criteria for Psychosomatic Research (DCPR) were proposed 20 years ago by an international group of investigators based on the recognition that a wide body of evidence has accumulated in psychosomatic medicine related to concepts of quality of life, stressful life events, somatization, and personality disorders [9] (online suppl. table 1; for all online suppl. material, see www.karger.com/doi/10.1159/000430788). The DCPR rationale was to expand the traditional domains of the disease model by translating psychosocial variables that derived from psychosomatic research into operational tools $[10,11]$. The DCPR are a set of 12 psychosomatic syndromes whose prognostic role in the development, course, and outcome of physical diseases, regardless of their 'organic' or 'functional' nature, was documented by a large body of literature. They include the diagnostic criteria for abnormal illness behavior (AIB; disease phobia, thanatophobia, health anxiety, illness denial), the various modalities of somatization (persistent somatization, functional somatic symptoms secondary to a psychiatric disorder, conversion symptoms, anniversary reaction), irritability (irritable mood, type A behavior), demoralization, and alexithymia.

The DCPR have undergone extensive validation [7, 12], as summarized in a monograph that also included a semistructured clinical interview for their assessment [13]. They have been used in several medical and psychiatric settings, also in different cultures such as Lithuania
[14], Japan [15], and India [16]. This diagnostic system enables clinicians to identify psychological problems in medical patients to a much greater extent than the DSM classification and also provides clinicians with information on specific psychological factors affecting a prevalent number of patients suffering from a given group of medical illnesses [17].

The aim of this paper is to provide a review of relevant studies which best display how the DCPR system can be clinically useful for (1) subtyping medical patients, (2) identifying subthreshold or undetected syndromes, (3) evaluating the burden of medical syndromes, and (4) predicting treatment outcomes and identifying risk factors.

\section{Methods}

Published reports involving the use of the DCPR were identified by searching in Medline, PsychInfo and Web of Science, from 1995 through to January 2015. A manual search of the literature was also performed for further studies not yet identified. Studies concerned with the clinical utility of the DCPR were selected and reviewed according to the above-mentioned issues.

\section{Results}

The initial search strategies yielded 184 published reports for potential inclusion in the review. Only those studies which best showed the clinical utility of the DCPR were selected.

\section{Subtyping Medical Patients}

DCPR syndromes may be used for subtyping patients who are characterized by distinct psychological profiles, even though they are lumped together because they present with the same diagnosis. The basic assumption of this approach is that clinical manifestations of a given medical or psychiatric diagnosis may display substantial interindividual differences in prognostic and therapeutic terms of relative weights assigned to different, though interdependent factors.

Data from a cross-sectional assessment using both DSM-IV and DCPR in 1,560 patients recruited from different medical settings were submitted to cluster analysis [18] and yielded 3 clusters: 1 comprised one third of the patients and was characterized by a low rate of DSM-IV disorders (5-8\%) and no DCPR syndromes, 1 comprised one quarter of the patients and was characterized by the presence of DCPR irritability only, and 1 cluster comprised about $40 \%$ of the patients and was characterized by 
the predominance of DCPR somatization and DSM mood/anxiety disorders. This study showed that the DCPR system allows to represent more adequately clinical reality by overcoming two restrictive dimensions of DSM, namely the hierarchical rule (because somatization syndromes co-occurred with axis I disorders and can be found also in patients with medical disorders that have known organic causes) and the exclusion of subthreshold symptomatology (because demoralization, AIB, and irritability are not mentioned in the DSM and not covered by the health-related worries indicated in the DSM-5based somatic symptom disorder).

Consistent findings from cluster analyses were obtained by analyzing samples with different psychiatric and medical disorders. In a study of 198 medical patients with major depressive disorder, Guidi et al. [19] identified two clusters characterized by DCPR somatization (and alexithymia) and DCPR irritability (and DSM anxiety disorders), respectively. Notably, patients with DCPR AIB (25\%) were equally represented in both clusters. Similarly, among medical patients diagnosed with DCPR demoralization [20], about one quarter of the sample was characterized by comorbid DSM-IV mood disorders (mainly major depressive disorder), whereas in two additional clusters, DSM-IV somatoform (and adjustment) and anxiety disorders were predominant; further, about one third of the sample was characterized by the lack of any comorbid DSM disorder, thus highlighting the importance of identifying and subtyping demoralization in the setting of medical disease, as advocated also in major depression.

In medical patients, the DCPR category of alexithymia was found in about one third of the cases associated with a comorbid DSM-IV mood or anxiety disorder, in another third with various forms of somatization and AIB, and in the remaining sample with no psychiatric comorbidity [21]. The joint use of DSM-IV and DCPR diagnostic criteria yielded more specific and detailed identification of alexithymic features together with distinct distress manifestations.

In a study on anorexia nervosa (AN) patients [22], mostly subjects with restricted-type AN, almost half of the patients had DCPR illness denial, associated with lower intensity of problems in body mass index, eating behaviors, and beliefs. A quarter of the restricted-type AN patients had high global severity in terms of body mass index, behaviors, thoughts, and DCPR conditions (high rates of alexithymia, demoralization, health anxiety, and irritable mood, ranging from 85 to $100 \%$ ). The last quarter was formed by purging-type AN patients and was characterized by severe illness and DCPR syndromes of somatization and demoralization.

The clinically relevant presence of DCPR illness denial was found also in medical patients with agoraphobia without panic disorder who, compared to patients with panic disorder and agoraphobia, were highly characterized by DCPR illness denial [23]. If panic attacks have not taken place, agoraphobic fears tend to appear natural to the individual and are often highly rationalized; patients are thus unlikely to seek treatment. Further, agoraphobia without panic was also significantly related to the poor insight into the role of psychological states in the production of somatic symptoms which occurs in DCPR persistent somatization and anniversary reactions. Without the use of a careful clinical evaluation, as the interview for DCPR, these features may be undetected and patients undertreated.

In patients with different levels of hypertension [24], cluster analysis revealed an association of three distinct subgroups (i.e. 'anxiety/depression', 'alexithymia', and 'somatization') with different levels of hypertension. In particular, patients with moderate-to-severe hypertension were more likely to belong to the 'anxiety/depression' and the 'alexithymia' subgroups, whereas the 'somatization' cluster was found to be associated with isolated systolic hypertension. These results provided further support to the importance of identifying and monitoring the clinical course of hypertensive patients characterized by specific clinical and subclinical psychological profiles.

In smaller samples not allowing a reliable cluster analysis, data fully supported the clinical utility of DCPR in yielding distinct psychological profiles of patients presenting with a given medical or psychiatric condition. In a controlled study [25], patients with primary aldosteronism were compared with patients with essential hypertension and matched normotensive subjects. A significantly higher prevalence was found for anxiety disorders (according to DSM-IV) among patients with primary aldosteronism compared to others. Irritable mood, as assessed by the DCPR, was significantly more frequent in both primary aldosteronism and essential hypertension compared with controls, whereas demoralization and persistent somatization did not differentiate between the subgroups. Clinical assessment of the DCPR diagnostic rubrics judged as relevant to endocrine disease yielded the identification of distinct psychological features of patients with primary aldosteronism, suggesting a role of mineralocorticoid regulatory mechanisms in clinical situations concerned with anxiety/irritability. 
The DCPR were also administered in a controlled study aimed at assessing subthreshold symptomatology in cyclothymic outpatients using a broad assessment strategy geared to subclinical signs [26]. Among cyclothymic patients, a comorbid DSM-IV diagnosis occurred in about two thirds of the cases and involved an anxiety disorder in most of the cases. A DCPR syndrome was found in the majority of cases (76\%), particularly demoralization, irritable mood, and other diagnostic rubrics concerned with DCPR somatization processes. A few DSM and DCPR diagnoses were reported also in the group of matched control subjects. Thus, the DCPR supplemented clinical data obtained by traditional diagnostic assessment tools and provided a detailed clinical configuration of cyclothymic disturbances, with important prognostic and clinical implications.

Subtyping patients based on the DCPR syndromes address two interconnected aspects of clinical utility. On the one hand, subtyping helps clinicians to tailor different decision and interventions based on the psychological characteristics of a given individual, according to the clinimetric perspective of macroanalysis [27]. On the other hand, subtyping helps researchers in identifying the core elements that make different patients really homogeneous, beyond macrocategories of personality constructs or psychopathological disorders or medical illnesses [28].

\section{Identifying Subthreshold or Undetected Syndromes}

Another field where the DCPR system has shown its clinical utility is the identification of psychopathological conditions that either have subclinical manifestations not meeting diagnostic criteria for psychopathology or are completely ignored by traditional nosography. So far, research data have been published on five undetected or subthreshold conditions not detected by traditional psychiatric classification: somatization, demoralization, irritability, anniversary reaction, and allostatic load (AO) syndrome.

Somatization [29] is a very frequent clinical condition both in primary care and specialty settings that cuts medical and psychiatric categories [30], has a considerable socioeconomic burden in terms of direct and indirect costs [31], and whose clinical reality is not adequately represented by DSM-5 [17]. For example, in patients with functional gastrointestinal disorders and myocardial infarction (MI) [32], 43\% had DCPR somatization syndromes, while only $16 \%$ had 1 of the DSM-IV somatoform disorders, and $82 \%$ had at least 1 DCPR somatization syndrome but no somatoform disorder, while only $14 \%$ had 1 somatoform disorder but no DCPR somatization.
Demoralization [33] is a subclinical form of mood disorder which both frequently precedes the onset of medical diseases and is triggered by the experience of illness $[34,35]$. According to de Figueiredo [36, 37], demoralization results from the convergence of psychological distress (helplessness and hopelessness) and subjective incompetence (the idea to be unable to cope with a stressful situation). In 807 consecutive outpatients recruited from different medical settings, $44 \%$ of the patients meeting the DSM-IV criteria for major depression were not found positive to DCPR criteria of demoralization, whereas $69 \%$ of the patients with DCPR demoralization did not meet the DSM criteria for major depression [38], thus suggesting the conclusion that demoralization and major depression are related but distinct constructs and should be diagnosed separately.

Similar conclusions can be reached when irritability is considered. Irritable mood (one of the two DCPR irritability syndromes) may be part of other psychiatric syndromes, especially major depressive disorder, but may also be an independent mood state [39]. In fact, $67 \%$ of medical patients with major depression were not classified as irritable, and $77 \%$ of patients with irritable mood did not satisfy the criteria for major depression [40], thus supporting the need for diagnostic criteria for assessing separately irritable mood (DCPR) and depression (DSM). Indeed, irritability together with demoralization may be a serious risk factor for these patients as it often precedes episodes of MI and is associated with a worse clinical outcome [41]. Type A behavior is the other DCPR irritability syndrome that, even though is not present in any version of the DSM, is very familiar to clinicians, both clinical psychologists and cardiologists. The DCPR type A behavior syndrome was more prevalent in patients with (36\%) than without (11\%) cardiovascular disorders (CV) [42]. The fact that only one third of the CV patients showed this behavioral pattern indicates that the expectation of finding it in every patient with CV disease is unrealistic, whereas it may mark a subtype of illness. Furthermore, DCPR irritability and demoralization syndromes were found in a prevalence ranging from 40 to $56 \%$ in patients with a recent MI, while DSM depressive and anxiety disorders ranged from 11 to $17 \%$ [43]. It is interesting to note that type A behavior may share some features with hypomania, cyclothymia, and hyperthymic temperament which frequently results in an overoptimistic view of one's own ability to cope with a stressful situation (as is a life-threatening disease) and in the minimization of vulnerability to future difficulties (e.g. medical complications) [44]. 
Anniversary reaction is often unrecognized because of the lack of awareness of the patient (and the negligence of the clinician) of the association between his or her symptoms and the occurrence of the anniversary of a meaningful event in his or her life. As the prevalence of this syndrome in medical patients is unknown, in the first large investigation by using the DCPR system, anniversary reaction was found in $54(3.6 \%)$ out of 1,498 medical patients, mostly women (76\%) [45]. It is remarkably that no patient with anniversary reaction was diagnosed with DSM-IV conversion disorder while co-occurrence with DCPR conversion symptoms was found in $20 \%$ of them. The DCPR assessment of anniversary reaction and conversion phenomena was based on Engel's [46] criteria that encompassed positive elements of identification (ambivalence; histrionic personality features; precipitation of symptoms by psychological stress, of which association the patient might be unaware; history of similar physical symptoms experienced by the patient or observed in someone else or wished on someone else) and did not exclude coexisting medical illness.

Finally, the presence of psychosomatic symptoms according to the DCPR classification has been included in the clinimetric criteria for the determination of $\mathrm{AO}$, a state due to the cumulative interactions between life events and chronic life stressors that, by exceeding the individual resources, may constitute a danger to health [47]. Among patients with atrial fibrillation, $\mathrm{AO}$ was significantly associated with increased psychological distress and greater prevalence of the DCPR syndromes of health anxiety and demoralization [48]. In outpatients with chronic cardiovascular illness [49], AO was related to poorer psychosocial functioning, higher rates of psychopathology (mainly mood and anxiety disorders), and emotional burden with DCPR syndromes of AIB, somatization, irritability, and demoralization. Furthermore, DCPR demoralization was found to be an independent predictor of $\mathrm{AO}$.

\section{Evaluating the Burden of Medical Illness}

It is known that psychopathology and psychosocial distress increase the burden of illness in the medically ill [50]. Less is known on the extent to which subclinical psychopathology and psychosocial stress contribute to the global burden of disease. In this regard, the DCPR system has been used in different medical settings including oncology, dermatology, consultation-liaison psychiatry, cardiology, and endocrinology.

In a convenience sample of 105 women with a recent diagnosis of breast cancer (within 18 months) and a good level of functioning [51], the impairment of the quality of life was more severe in patients meeting the DCPR for demoralization, irritability, and alexithymia, namely those patients who were living their disease with a sense of inadequacy and failure, irritating feelings, or marked difficulty to express their emotions.

In a large consecutive group of tertiary-care patients with skin disorders, having both DCPR and DSM-IV diagnoses significantly worsened the overall somatic and psychological health status compared to patients with either DCPR or DSM-IV syndromes and doubled the overall burden of disease compared to patients without DCPR and DSM-IV diagnoses [52]. Another study was performed on patients with a very high level of psychopathology such as those hospitalized for a medical problem and referred to psychiatric consultation because of suspected psychiatric comorbidity (confirmed in $89 \%$ of them) [53]. After controlling for covariates, the DCPR syndromes (particularly demoralization and health anxiety) and high levels of somatization independently predicted psychosocial maladaptation over and above DSMIV diagnoses.

In line with previous findings, patients who received heart transplantations were assessed for evaluating the relative weight of major depressive disorder and DCPR demoralization in their quality of life and subjective wellbeing [41]. Patients with DCPR demoralization had more impairment in all dimensions of their quality of life (physical, psychological, social, and environmental); lower psychological well-being, particularly environmental mastery and self-acceptance; and severer anxiety and depressive symptoms, somatization, and hostility.

The joint use of the DSM-IV and DCPR diagnostic assessment tools also allowed to elicit both psychiatric and psychological conditions relevant to endocrine disease. Psychological distress was found to persist also in endocrine patients who were cured or in remission [54], with a high prevalence of DCPR irritable mood, demoralization, and persistent somatization as well as DSM-IV mood and anxiety disorders. In a controlled study of outpatients with pituitary disorders in remission after appropriate treatment, significant differences were found in psychological distress compared to matched healthy control subjects [55]. Patients with pituitary disease displayed a higher prevalence of DCPR syndromes (i.e. irritable mood, demoralization, and persistent somatization) and psychiatric diagnoses (according to DSM-IV) compared to controls, thus indicating difficulties in obtaining full recovery in patients treated for endocrine disorders and the need for improving the level of remission 
in such patients by introducing additional therapeutic strategies.

These studies confirm that the DCPR assessment, alone or together with the traditional psychiatric nosography, gives clinicians the possibility to identify the patients at a higher risk of distress because of their elevated burden of disease.

\section{Predicting Treatment Outcome and Identifying Risk \\ Factors}

Understanding the psychosocial variables influencing the treatment outcome is particularly useful in clinical practice because it may help the identification of patients who are more likely to not respond to standard medical treatment or are more at risk for severe disease episodes. Interesting findings were obtained by performing a broad psychological assessment, including the use of the DCPR, in gastroenterology and cardiology.

In patients with moderate-to-severe functional gastrointestinal disorder, nonresponse to treatment was significantly and independently predicted by the DCPR syndromes of alexithymia and persistent somatization, while improvement after treatment was predicted by DCPR health anxiety [56]. Furthermore, in patients with recent MI in cardiac rehabilitation, CV events (death, reinfarction, and angina pectoris) after 2 years were associated to the presence of one or more DCPR syndromes, age, psychological distress, and poor well-being [57].

In cardiology, two independent case-control studies found that the DCPR demoralization syndrome significantly characterized the prodromal phase of $\mathrm{CV}$ events in patients with recent MI [43] and acute coronary heart disease [58]. However, in a follow-up study of 2.5 years, no direct association between demoralization and poor cardiac outcomes (reinfarction, unstable angina, angioplasty, bypass surgery, heart failure, and cardiac death) was found when controlled for by dysthymia [59]. Demoralization also characterized patients with heart transplantation [41]. In particular, it was related to impairments in physical, psychological, social, and environmental quality of life and in psychological well-being. The co-occurrence of a major depressive episode did not alter this pattern of associations, whereas the addition of demoralization to major depressive disorder resulted in decreased psychosocial functioning. In a survival analysis after 6 years, after controlling for multiple medical variables (cardiac events, renal insufficiency, medications, diabetes, and severity of cardiopathy), high levels of hostility (a clinically relevant component of the DCPR irritability cluster) were found to independently predict survival status [60].

\section{Conclusion}

A diagnostic tool has clinical utility if it may help clinicians to recognize mechanisms and clinical conditions underlying symptom presentation, enable the identification of unrecognized problems, enhance communication among clinicians and in the doctor-patient relationship, and influence the multiple decisions and outcome [1]. As shown in this review, the clinical utility of DCPR may substantially add to personalized health care, i.e. to the tailoring of the diagnosis and the management and treatment of each patient to his or her individual characteristics [61]. As the personalized approach to individual patients has until now focused mainly on identifying biological markers that could predict the disease course, treatment response, and risk of side effects, DCPR are particularly helpful in fields where biological markers are not available as psychiatry [62] or psychosomatic medicine $[61,63]$.

On the one hand, in the setting of clinical psychiatry, there is an increasing awareness that the DSM system captures only a narrow part of the information necessary for the clinical utility process [64]. The traditional classification in psychiatry does not include relevant information of clinical utility such as patterns of psychosocial factors, coping strategies, burden of illness, effects of comorbid conditions, responses to previous treatments, and other clinical distinctions which are likely to influence the course, therapeutic response and outcome of a given illness and that demarcate major prognostic and therapeutic differences among patients who otherwise seem to be deceptively similar since they share the same psychiatric diagnosis $[65,66]$. In particular, the new DSM-5 category of somatic symptom disorder seems to neglect important features concerning the psychological factors affecting medical conditions and AIB. The 12 DCPR syndromes may broaden the clinician's perspective on patient problems by providing additional clinical information which does not find any space in the traditional psychiatric classification [17] and therefore may be suggested as operative tools in psychosomatic-based outpatient services and clinics [67]. An example of the clinical application of the DCPR and DSM-5 criteria to two clinical cases is shown in the online supplementary table 2 .

On the other hand, in the medical setting, the traditional biomedical disease model cannot explain how recognition, presentation, and outcome of a disease may be largely influenced by individual responses to symptoms [11]. Many years ago, Feinstein [68] already urged clini- 
cians to develop alternative intellectual models to study the clinical phenomena directly, to specify the importance of different types of clinical data, and to create appropriate systems of taxonomy for classifying the information that would articulate the clinical process and use the results for quantified analyses for enhancing the process of clinical decision making.

The lesson from the literature on DCPR points to the clinical utility of expanding the traditional symptombased psychiatric taxonomy or biomedical disease model.
Future challenges concern the joint use of the DCPR and neurobiological investigations to fill the gap between biomarkers and clinical judgment $[27,61]$ and the application of specific treatments to the DCPR syndromes for improving quality of life and clinical outcomes.

\section{Disclosure Statement}

None of the authors has any conflicts of interest to declare.

\section{References}

1 Barron J (ed): Making Diagnosis Meaningful. Enhancing Evaluation and Treatment of Psychological Disorders. Washington, American Psychological Association, 1998.

2 Haynes SN, Leisen MB, Blaine DD: Design of individualized behavioral treatment programs using functional analytic clinical case models. Psychol Assess 1997:9:334-348.

3 Murphy KR, Davidshofer CO: Psychological Testing. Principles and Applications, ed 2. Englewood Cliffs, Prentice Hall, 1991.

4 Foster SL, Cone JD: Validity issues in clinical assessment. Psychol Assess 1995;7:248-260.

5 Schröder A, Fink P: The proposed diagnosis of somatic symptom disorders in DSM-V: two steps forward and one step backward? J Psychosom Res 2010;68:95-96.

6 Sirri L, Fava GA, Wise TN: Psychiatric classification in the setting of medical disease: comparing the clinical value of different proposals. J Psychosom Res 2011;70:493-495.

7 Sirri L, Fava GA: Diagnostic criteria for psychosomatic research and somatic symptom disorders. Int Rev Psychiatry 2013;25:19-30.

8 Van der Feltz-Cornelis CM, van Balkom AJLM: The concept of comorbidity in somatoform disorder - a DSM-V alternative for the DSM-IV classification of somatoform disorders. J Psychosom Res 2010;68:97-99.

9 Fava GA, Freyberger HJ, Bech P, Christodoulou G, Sensky T, Theorell T, Wise TN: Diagnostic criteria for use in psychosomatic research. Psychother Psychosom 1995;63:1-8.

10 Sirri L, Fabbri S, Fava GA, Sonino N: New strategies in the assessment of psychological factors affecting medical conditions. J Pers Assess 2007;89:216-228.

11 Fava GA, Sonino N, Wise TN (eds): The Psychosomatic Assessment. Strategies to Improve Clinical Practice. Basel, Karger, 2012.

12 Porcelli P, Rafanelli C: Criteria for Psychosomatic Research (DCPR) in the medical setting. Curr Psychiat Rep 2010;12:246-254.

13 Porcelli P, Sonino N (eds): Psychological Factors Affecting Medical Conditions. A New Classification for DSM-V. Basel, Karger, 2007.
14 Beresnevaité M, Taylor G J, Bagby RM: Assessing alexithymia and type A behavior in coronary heart disease patients: a multimethod approach. Psychother Psychosom 2007;76:186-192.

15 Fukunishi I, Hosaka T, Aoki T, Azekawa T, Ota A, Miyaoka H: Criterion-related validity of diagnostic criteria for alexithymia in a general hospital psychiatric setting. Psychother Psychosom 1996;65:82-85.

16 Chaturvedi SK, Goswami K: Feasibility of Diagnostic Criteria for Psychosomatic Research in India: a pilot qualitative evaluation. Psychother Psychosom 2012;81:320-321.

17 Guidi J, Rafanelli C, Roncuzzi R, Sirri L, Fava GA: Assessing psychological factors affecting medical conditions: comparison between different proposals. Gen Hosp Psychiatry 2013; 35:141-146.

18 Fava GA, Guidi J, Porcelli P, Rafanelli C, Bellomo A, Grandi S, Grassi L, Mangelli L, Pasquini P, Picardi A, Quartesan R, Rigatelli M, Sonino N: A cluster analysis-derived classification of psychological distress and illness behavior in the medically ill. Psychol Med 2012; 42:401-407.

19 Guidi J, Fava GA, Picardi A, Porcelli P, Bellomo A, Grandi S, Grassi L, Pasquini P, Quartesan R, Rafanelli C, Rigatelli M, Sonino $\mathrm{N}$ : Subtyping depression in the medically ill by cluster analysis. J Aff Dis 2011;132:383388.

20 Rafanelli C, Guidi J, Gostoli S, Tomba E, Porcelli P, Grandi S: Subtyping demoralization in the medically ill by cluster analysis. Eur J Psychiat 2013;27:7-17.

21 Porcelli P, Guidi J, Sirri L, Grandi S, Grassi L, Ottolini F, Pasquini P, Picardi A, Rafanelli C, Rigatelli M, Sonino N, Fava GA: Alexithymia in the medically ill. Analysis of 1190 patients in gastroenterology, cardiology, oncology and dermatology. Gen Hosp Psychiatry 2013;35: 521-527.

22 Abbate-Daga G, Delsedime N, Nicotra B, Giovannone C, Marzola E., Amianto F, Fassino S: Psychosomatic syndromes and anorexia nervosa. BMC Psychiatry 2013;13:14.
23 Fava GA, Porcelli P, Rafanelli C, Mangelli L, Grandi S: The spectrum of anxiety disorders in the medically ill. J Clin Psychiatry 2010;71: 910-914.

24 Rafanelli C, Offidani E, Gostoli S, Roncuzzi R: Psychological correlates in patients with different levels of hypertension. Psychiat Res 2012;198:154-160.

25 Sonino N, Ruini C, Navarrini C, Ottolini F Sirri L, Paoletta A, Fallo F, Boscaro M, Fava GA: Psychosocial impairment in patients treated for pituitary disease: a controlled study. Clin Endocrinol 2007;67:719-726.

26 Tomba E, Rafanelli C, Grandi S, Guidi J, Fava GA: Clinical configuration of cyclothymic disturbances. J Affect Disord 2012;139:244249.

27 Fava GA, Guidi J, Grandi S, Hasler G: The missing link between clinical states and biomarkers in mental disorders. Psychother Psychosom 2014;83:136-141.

28 Fava GA, Tossani E, Bech P, Berrocal C, Chouinard G, Csillag C, Wittchen HU, Rief $\mathrm{W}$ : Emerging clinical trends and perspectives on comorbid patterns of mental disorders in research. Int J Meth Psychiat Res 2014; 23(suppl 1):92-101.

29 Lipowski ZJ: Somatization: the experience and communication of psychological distress as somatic symptoms. Psychother Psychosom 1987;47:160-167.

30 Kroenke K: Patients presenting with somatic complaints: epidemiology, psychiatric comorbidity, and management. Int J Meth Psychiat Res 2003;12:34-43.

31 Konnopka A, Kaufmann C, König HH, Heider D, Wild B, Szecsenyi J, Herzog W, Heinrich S, Schaefert R: Association of costs with somatic symptom severity in patients with medically unexplained symptoms. J Psychosom Res 2013;75:370-375.

32 Mangelli L, Bravi A, Fava GA, Ottolini F, Porcelli P, Rafanelli C, Rigatelli M, Sonino N: Assessing somatization with various diagnostic criteria. Psychosomatics 2009;50:38-41. 
33 Clarke DM, Kissane DW: Demoralization: its phenomenology and importance. Austr NZ J Psychiatry 2002;36:733-742.

34 Slavney PR: Diagnosing demoralization in consultation psychiatry. Psychosomatics 1999; 40:325-329.

35 Tecuta L, Tomba E, Grandi S, Fava GA: Demoralization: a systematic review on its clinical characterization. Psychol Med 2015;45: 673-691.

36 De Figueiredo JM: Demoralization and psychotherapy: a tribute to Jerome D. Frank, MD, PhD (1909-2005). Psychother Psychosom 2007;76:129-133.

37 De Figueiredo JM: Distress, demoralization and psychopathology: diagnostic boundaries. Eur J Psychiatry 2013;7:61-73.

38 Mangelli L, Fava GA, Grandi S, Grassi L, Ottolini F, Porcelli P, Rafanelli C, Rigatelli M, Sonino N. Assessing demoralization and depression in the setting of medical disease. J Clin Psychiatry 2005;66:391-394.

39 Snaith RP, Taylor CM: Irritability. Br J Psychiatry $1985 ; 147: 127-136$

40 Mangelli L, Fava GA, Grassi L, Ottolini F, Paolini S, Porcelli P, Rafanelli C, Rigatelli M, Sonino N: Irritable mood in Italian patients with medical disease. J Nerv Ment Dis 2006; 194:226-228.

41 Grandi S, Sirri L, Tossani E, Fava GA: Psychological characterization of demoralization in the setting of heart transplantation. J Clin Psychiatry 2011;72:648-654.

42 Sirri L, Fava GA, Guidi J, Porcelli P, Rafanelli C, Bellomo A, Grandi S, Grassi L, Pasquini P, Picardi A, Quartesan R, Rigatelli M, Sonino $\mathrm{N}$ : Type A behavior: a reappraisal of its characteristics in cardiovascular disease. Int J Clin Pract 2012;66:854-861.

43 Ottolini F, Modena MG, Rigatelli M: Prodromal symptoms in myocardial infarction. Psychother Psychosom 2005;74:323-327.

44 Wang Y, Terao T, Hoaki N, Goto S, Tsuchiyama K, Iwata N, Yoshimura R, Nakamura J: Type A behavior pattern and hyperthymic temperament: possible association with bipolar IV disorder. J Affect Dis 2011;133:22-28.

45 Porcelli P, Fava GA, Rafanelli C, Bellomo A, Grandi S, Grassi L, Pasquini P, Picardi A, Quartesan R, Rigatelli M, Sonino N: Anniversary reactions in medical patients. J Nerv Men Dis 2012;200:603-606.
46 Engel GL: Conversion symptoms; in MacBryde CM, Blacklow RS (eds): Signs and Symptoms. Philadelphia, Lippincott, 1970, pp 650-669.

47 Fava GA, Guidi J, Semprini F, Tomba E, Sonino N: Clinical assessment of allostatic load and clinimetric criteria. Psychother Psychosom 2010;79:280-284.

48 Offidani E, Rafanelli C, Gostoli S, Marchetti G, Roncuzzi R: Allostatic overload in patients with atrial fibrillation. Int J Cardiol 2012;165: 375-376.

49 Porcelli P, Laera D, Mastrangelo D, Di Masi A: Prevalence of allostatic overload syndrome in patients with chronic cardiovascular disease. Psychother Psychosom 2012;81:375.

50 National Collaborating Centre for Mental Health: Depression in Adults with a Chronic Physical Health Problem. London, Royal College of Psychiatrists, 2010.

51 Grassi L, Rossi E, Sabato S, Cruciani G, Zambelli M: Diagnostic criteria for psychosomatic research and psychosocial variables in breast cancer patients. Psychosomatics 2004;45: 483-491.

52 Picardi A, Pasquini P, Abeni D, Fassone G Mazzotti E, Fava GA: Psychosomatic assessment of skin diseases in clinical practice. Psychother Psychosom 2005;74:315-322.

53 Porcelli P, Bellomo A, Quartesan R, Altamura M, Iuso S, Ciannameo I, Piselli M, Elisei S: Psychosocial functioning in consultation-liaison psychiatry patients: influence of psychosomatic syndromes, psychopathology and somatization. Psychother Psychosom 2009;78: 352-358.

54 Sonino N, Navarrini C, Ruini C, Ottolini F, Paoletta A, Fallo F, Boscaro M, Fava GA: Persistent psychological distress in patients treated for endocrine disease. Psychother Psychosom 2004;73:78-83.

55 Sonino N, Tomba E, Genesia ML, Bertello C, Mulatero P, Veglio F, Fava GA, Fallo F: Psychological assessment of primary aldosteronism: a controlled study. J Clin Endocrinol Metab 2011;96:E878-E883.

56 Porcelli P, De Carne M, Todarello O: Prediction of treatment outcome of patients with functional gastrointestinal disorders by the Diagnostic Criteria for Psychosomatic Research. Psychother Psychosom 2004;73:166173.
57 Rafanelli C, Roncuzzi R, Finos L, Tossani E, Tomba E, Mangelli L, Urbinati S, Pinelli G, Fava GA: Psychological assessment in cardiac rehabilitation. Psychother Psychosom 2003; 72:343-349.

58 Rafanelli C, Roncuzzi R, Milaneschi Y, Tomba E, Colistro MC, Pancaldi LG, Di Pasquale G: Stressful life events, depression and demoralization as risk factors for acute coronary heart disease. Psychother Psychosom 2005; 74:179-184.

59 Rafanelli C, Milaneschi Y, Roncuzzi R, Pancaldi LG: Dysthymia before myocardial infarction as a cardiac risk factor at 2.5-year follow-up. Psychosomatics 2010;51:8-13.

60 Sirri L, Potena L, Masetti M, Tossani E, Magelli C, Grandi S: Psychological predictors of mortality in heart transplanted patients: a prospective, 6-year follow-up study. Transplantation 2010;89:879-886.

61 Evers AWM, Gieler U, Hasenbring MI, van Middendorp H: Incorporating biopsychosocial characteristics into personalized healthcare: a clinical approach. Psychother Psychosom 2014;83:148-157.

62 Rafanelli C, Sirri L, Grandi S, Fava GA: Is depression the wrong treatment target for improving outcome in coronary artery disease? Psychother Psychosom 2013;82:285-291.

63 Zimmerman M: Screening for bipolar disorder: confusion between case-finding and screening. Psychother Psychosom 2014;83:259-262.

64 Vanheule S, Desmet M, Meganck R, Inslegers R, Willemsen J, De Schryver M, Devisch I: Reliability in psychiatric diagnosis with the DSM: old wine in new barrels. Psychother Psychosom 2014;83:313-314.

65 Sirri L, Fava GA, Sonino N: The unifying concept of illness behavior. Psychother Psychosom 2013;82:74-81.

66 Fava GA, Rafanelli C, Tomba E: The clinical process in psychiatry: a clinimetric approach. J Clin Psychiatry 2012;73:177-184.

67 Sonino N, Peruzzi P: A psychoneuroendocrinology service. Psychother Psychosom 2009; 78:346-351.

68 Feinstein AR: Clinical Judgment. Baltimore, Williams \& Wilkins, 1967. 